\title{
MicroRNA-22 contributes to dexamethasone-induced osteoblast differentiation inhibition and dysfunction through targeting caveolin-3 expression in osteoblastic cells
}

\author{
PENG LI ${ }^{1}$, WEIWEI MAO ${ }^{2}$, SHUAI ZHANG ${ }^{1}$, LIANG ZHANG ${ }^{1}$, ZHIRONG CHEN $^{1}$ and ZHIDONG LU ${ }^{1}$ \\ ${ }^{1}$ Department of Orthopedics, General Hospital of Ningxia Medical University, Yinchuan, Ningxia 750004; \\ ${ }^{2}$ Clinical Skill Center of Yinchuan First People's Hospital, Yinchuan, Ningxia 750001, P.R. China
}

Received May 30, 2020; Accepted October 30, 2020

DOI: $10.3892 /$ etm.2021.9767

\begin{abstract}
Osteoporosis is a common complication of long-term use of glucocorticoids (GCs) characterized by the loss of bone mass and damage of the microarchitecture as well as osteoblast dysfunction. Previous studies have demonstrated that microRNA-22 (miR-22) is the negative modulator of osteogenesis that may target caveolin-3 (CAV3), which has been reported to enhance bone formation and inhibit the progression of osteoporosis as well as apoptosis. The present study aimed to investigate whether miR-22 may be involved in dexamethasone (DEX)-induced inhibition of osteoblast differentiation and dysfunction by regulating CAV3 expression. Reverse transcription-quantitative PCR (RT-qPCR) was performed to measure the expression of miR-22 and western blotting was performed to determine protein levels. The results demonstrated that miR-22 expression was upregulated in DEX-treated osteoblastic cells compared with the control group. In addition, miR-22 mimic aggravated, whereas miR-22 inhibitor mitigated DEX-induced damage in osteoblastic cells compared with the control groups. Additionally, CAV3 was identified as the target of miR-22 in osteoblasts using RT-qPCR, western blotting and dual-luciferase reporter gene assay analysis. The results also demonstrated that silencing of CAV3 blocked the beneficial effects of miR-22 inhibitor against DEX-induced cell damage and apoptosis in osteoblasts, as evidenced by the increased expression levels of cleaved caspase-3, Bax and alkaline phosphatase activity as well as decreased cell viability and Bcl-2 levels. Collectively, these results indicate a novel molecular mechanism by which miR-22 contributes to DEX-induced osteoblast dysfunction and apoptosis via the miR-22/CAV3 pathway.
\end{abstract}

Correspondence to: Dr Zhidong Lu, Department of Orthopedics, General Hospital of Ningxia Medical University, 804 Shengli South Road, Xingqing, Yinchuan, Ningxia 750004, P.R. China E-mail: zhidonglu@126.com

Key words: microRNA-22, caveolin-3, osteoblasts, apoptosis, dexamethasone

\section{Introduction}

Glucocorticoids (GCs) are one of the most widely used classes of drugs due to their remarkable anti-inflammation and immunosuppressive function and it is estimated that $1-2 \%$ of the population is undergoing long-term GC therapy worldwide (1-4). However, extensive GC therapy leads to various adverse effects, and one of the most severe of them is GC-induced osteoporosis (GIO), characterized by systemic damage of the bone mass and the microarchitecture that induces fragility fractures (5). Previous studies have demonstrated that the lack of balance between bone formation and resorption induced by osteoblast dysfunction, such as inhibited proliferation and differentiation and enhanced apoptosis of osteoblasts, is involved in GIO (5-7). However, the underlying mechanism of GIO has not been fully elucidated.

MicroRNAs (miRs) are a class of small ( 22 nucleotides), highly conserved noncoding RNAs that post-transcriptionally regulate the expression of their target genes and exert pivotal roles in multiple diseases, including chronic lymphocytic leukemia, Huntington's disease and myocardial ischemia-reperfusion injury (8-10). An increasing number of studies have demonstrated that miRs are involved in the pathogenesis and development of osteoporosis (11-13). Shi et al (11) reported that miR-17/20a suppressed GC-induced osteoclast differentiation and dysfunction by targeting the expression of receptor activator of $\mathrm{NF} \kappa \mathrm{B}$ ligand (RANKL) in osteoblastic cells. In addition, Zhang et al (12) demonstrated that miR-221 participated in the process of osteoporosis by regulating the protein expression of runt-related transcription factor 2 and osteoblast differentiation. Notably, a previous study demonstrated that miR-22 is a negative modulator of osteogenesis (13). Additionally, oxidative stress triggered by excessive reactive oxygen species (ROS) production was reported to contribute to dexamethasone (DEX)-induced osteoporosis $(7,14,15)$, and miR-22 facilitated myocardial damage following ischemia/reperfusion insult and hypoxia/reoxygenation-induced dysfunction in cultured cardiomyocytes by inducing mitochondrial oxidative stress (9). However, whether miR-22 is involved in the development of DEX-induced osteoporosis by targeting the oxidative stress process remains to be determined. Notably, Zhang et al (16) demonstrated that 
caveolin-3 (CAV3) is a direct target of miR-22, containing two seed binding sites in its 3'-untranslated region (3'-UTR). As a member of the caveolin proteins (CAV1, 2 and 3) with six subsets, CAV3 is involved not only in the formation of caveolae, but also bone formation and osteoporosis progression $(14,17)$. Yang et al (14) demonstrated that upregulation of CAV3 enhances bone formation and inhibits the progression of osteoporosis by activating the Wnt signaling pathway in a rat model initially induced by means of ovariotomy, which suggested that CAV3 may be a potential biomarker and osteoporosis treatment target. Therefore, the present hypothesized that miR-22 may also be involved in osteoporosis progression by regulating the expression of CAV3.

Previous studies have demonstrated that GC-induced osteoblast apoptosis contributed to the development and progression of osteoporosis $(7,15)$. For instance, Gohel et al (18) reported that GC treatment resulted in osteoblast apoptosis in vivo and in vitro. In addition, upregulation of CAV3 prevented hypoxia or TNF- $\alpha$ treatment-induced cardiomyocyte apoptosis $(19,20)$.

The present study aimed to examine the expression of miR-22 in DEX-treated MC3T3-E1 cells and the effects of miR-22 mimic on DEX-induced osteoblast damage and apoptosis, and to investigate the effects of miR-22 on CAV3 expression in osteoblasts to determine whether miR-22 may be involved in DEX-induced osteoblast dysfunction and apoptosis through the regulation of CAV3 expression.

\section{Materials and methods}

Cell culture and drug treatments. The murine osteoblastic MC3T3-E1 cell line was purchased from the American Type Culture Collection and cultured in $\alpha$-MEM (Gibco; Thermo Fisher Scientific, Inc.) containing 10\% FBS (Thermo Fisher Scientific, Inc.) and $1 \%$ penicillin-streptomycin (cat. no. 10378016; Gibco; Thermo Fisher Scientific, Inc.) at $37^{\circ} \mathrm{C}$ with $5 \% \mathrm{CO}_{2}$ and $95 \%$ air. DEX (cat. no. D4902; Sigma-Aldrich; Merck KGaA) was dissolved in absolute ethanol and the cells were incubated with $1 \mu \mathrm{M}$ DEX in the culture medium for $72 \mathrm{~h}$ (21). N-acetyl L-cysteine (NAC; cat. no. A9165; Sigma-Aldrich; Merck KGaA) and p53 inhibitor pifithrin- $\alpha$ (cat. no. P4359; Sigma-Aldrich; Merck KGaA) were dissolved in saline and DMSO, respectively. Cells were incubated with $1 \mathrm{mM}$ NAC or $20 \mu \mathrm{M}$ pifithrin- $\alpha$ for $48 \mathrm{~h}$ (10).

miR-22 mimic, inhibitor and small interfering RNA (siRNA) transfection. miR-22 mimic, inhibitor and siRNA $(200 \mu \mathrm{M})$ transfections were performed using $\mathrm{Xfect}^{\mathrm{TM}}$ RNA transfection reagent (cat. no. 631450; Takara Bio, Inc.). At $37^{\circ} \mathrm{C}$, osteoblastic MC3T3-E1 cells were firstly transfected with control or CAV3 siRNA for $24 \mathrm{~h}$ and then transfected with miR control or miR-22 inhibitor for another $24 \mathrm{~h}$. Immediately after miR control or miR-22 inhibitor transfection, cells were treated with DEX for $72 \mathrm{~h}$. The sequences were as follows: miR-22 mimic sense, 5'-AAGCUGCCAGUUGAAGAACUGU-3' and antisense, 5'-AGUUCUUCAACUGGCAGCUUUU-3'; miR mimic control sense, 5'-UUCUUCGAACGUGUCACGUTT-3' and antisense, 5'-ACGUGACACGUUCGGAGAATT-3'; miR-22 inhibitor, 5'-ACAGUUCUUCAACUGGCAGCUU-3' miR inhibitor control, 5'-CAGUACUUUUGUGUAGUACAA-3', CAV3 siRNA sense, 5'-GCAGCAACAUUAAGGUGGUTT-3' and antisense, 5'-ACCACCUUAAUGUUGCUGCTT-3' and control siRNA sense, 5'-UUCUUCGAACGUGUCACGUTT-3' and antisense, 5'-ACGUGACACGUUCGGAGAATT-3'.

MTT assay. Cell viability was evaluated by an MTT assay (10). Briefly, $250 \mathrm{mg}$ MTT (cat. no. M5655; Sigma-Aldrich; Merck KGaA) was dissolved in $50 \mathrm{ml}$ PBS to produce a $5 \mathrm{~g} / \mathrm{l}$ solution and filtered through a $0.22-\mu \mathrm{m}$ filter. A 48 -well plate was used to incubate cells at a density of $2 \times 10^{5}$ cells $/ \mathrm{ml}$ in $200 \mu \mathrm{l}$ culture media, and the cells were exposed to MTT $(0.5 \mathrm{~g} / \mathrm{l})$ for $2-4 \mathrm{~h}$ following drug treatments. The generated formazan crystals were dissolved in DMSO, and the absorbance at a wavelength of $550 \mathrm{~nm}$ was measured by a microplate reader (Tecan Group, Ltd.).

Alkaline phosphatase (ALP) activity assay. Osteoblastic MC3T3-E1 cells were cultured in a 96-well plate at a density of $1 \times 10^{6}$ cells $/ \mathrm{ml}$ in $100 \mu \mathrm{l} \alpha$-MEM with $10 \%$ FBS. Subsequently, $1 \%$ Triton $\mathrm{X}-100$ was added to the medium and centrifuged for $20 \min \left(14,000 \times \mathrm{g}, 4^{\circ} \mathrm{C}\right)$ to recover the supernatant. The change in absorbance at a wavelength of $405 \mathrm{~nm}$ was measured by the method previously described by Bowers and McComb (22).

Dual luciferase reporter assay. The wild-type 3'-UTR and the miR-22 'seed' mutant 3'-UTR of CAV3 were synthesized in vitro and cloned into the psiCHECK2 luciferase reporter plasmid (cat. no. C8021; Promega Corporation). Osteoblastic MC3T3-E1 cells were co-transfected with the psiCHECK-2 plasmid containing the wild-type or mutant CAV3 3'-UTR and miRNA control or miR-22 mimic using Xfect ${ }^{\mathrm{TM}}$ Transfection Reagent (cat. no. 631317; Takara Bio, Inc.) and Xfect ${ }^{\mathrm{TM}}$ RNA transfection reagent (cat. no. 631450; Takara Bio, Inc.) respectively. Cell lysates were collected $24 \mathrm{~h}$ after transfection, and reporter activity was determined by measuring firefly luciferase activity by a dual luciferase reporter system (Dual-Luciferase Reporter Assay System; cat. no. E1910; Promega Corporation) and normalized to Renilla luciferase activity.

Reverse transcription-quantitative PCR (RT-qPCR). TRIzol $^{\circledR}$ reagent (Invitrogen; Thermo Fisher Scientific, Inc.) was used to extract total RNA from osteoblasts, which was reverse-transcribed to generate cDNA using SuperScript ${ }^{\mathrm{TM}}$ II reverse transcriptase (Thermo Fisher Scientific, Inc.) with a specific stem-loop primer for miR-22 and miR-16 and oligodeoxythymidine for mRNAs. The temperature protocol used was $25^{\circ} \mathrm{C}$ for $10 \mathrm{~min}, 42^{\circ} \mathrm{C}$ for $1 \mathrm{~h}$ and finally $72^{\circ} \mathrm{C} 10 \mathrm{~min}$. qPCR was performed using a MiniOpticon Real-Time PCR Detection system (Bio-Rad Laboratories, Inc.). The following primer pairs were used for the qPCR: CAV3 (GenBank accession no. NM_007617) sense, 5'-TCAACGATACCAGCCACA AG-3' and antisense, 5'-ACACCGTCGAAGCTGTAGGT-3'; GAPDH (GenBank accession no. NM_017008) sense, 5'-TCT ACATGTTCCAGTATGACTC-3' and antisense, 5'-ACTCCA CGACATACTCAGCACC-3' (16); miR-22 sense, 5'-GGG GGAGCTGCCAGTTGAAG-3' and antisense, 5'-GTGCAG GGTCCGAGGT-3' (10) and miR-16 sense, 5'-GCCCCTTCG TCGTTAGA-3' and antisense, 5'-GTGCAGGGTCCGAGG T-3' (10). The reaction solution consisted of $5.0 \mu 1$ diluted cDNA, $0.2 \mu \mathrm{M} / 1$ of each paired primer, 1X SYBR-Green qPCR Mix buffer (Toyobo Life Science) and $4.9 \mu \mathrm{l}$ diethyl 
pyrocarbonate-treated water. The annealing temperature was set at $58-62^{\circ} \mathrm{C}$ and amplification was set at 40 cycles. The temperature range to detect the melting temperature of the PCR product was set between $60-95^{\circ} \mathrm{C}$. To determine the relative quantitation of gene expression, the comparative $\mathrm{Ct}$ (threshold cycle) method with arithmetic formula $\left(2^{-\Delta \Delta \mathrm{Cq}}\right)$ was used (23). mRNA levels and miRNA levels were normalized relative to the housekeeping gene GAPDH and miR-16, respectively.

Western blot analysis. Osteoblastic MC3T3-E1 cells proteins were lysed using cold RIPA buffer (Beyotime Institute of Biotechnology) containing 1\% Protease Inhibitor Cocktail (Thermo Fisher Scientific, Inc.) and BCA assays were used to calculate the protein concentrations. Subsequently, $30 \mu \mathrm{g}$ proteins per lane were separated using 10\% SDS-PAGE and transferred to PVDF membranes. Following blocking with non-fat dry milk dissolved in TBS containing $0.05 \%$ Tween-20 for $1-2 \mathrm{~h}$ at room temperature, the membranes were incubated with diluted primary antibodies against CAV3 (cat. no. sc-5310; Santa Cruz Biotechnology, Inc.), Bax (cat. no. ab32503; Abcam), Bcl-2 (cat. no. ab182858; Abcam), pro- and cleaved caspase-3 (cat. no. sc-56053; Santa Cruz Biotechnology, Inc.), p53 (cat. no. 10422-1-AP; ProteinTech Group, Inc.), p21 (cat. no. 27296-1-AP; ProteinTech Group, Inc.) or $\beta$-actin (cat. no. sc-81178; Santa Cruz Biotechnology, Inc.) in primary antibody dilution buffer (Beyotime Institute of Biotechnology) at $4{ }^{\circ} \mathrm{C}$ overnight, all at a dilution of $1: 1,000$. Following washing with TBST buffer $(0.05 \%$ Tween-20, $0.15 \mathrm{M} \mathrm{NaCl}$, $50 \mathrm{mM}$ Tris-HCI, $\mathrm{pH}$ 7.5), the membranes were incubated with a horseradish peroxidase-conjugated secondary antibody (cat. nos. SA00001-1 and SA00001-2; ProteinTech Group, Inc.) for $1-2 \mathrm{~h}$ at room temperature at a dilution of 1:2,000. The Enhanced Chemiluminescence Western Blotting Detection system (Santa Cruz Biotechnology, Inc.) and a GeneGnome HR scanner (SynGene Europe) were used to visualize the immunoreactive proteins and detect chemiluminescent signals from the membranes using GeneSnap software version 7.12 (Syngene).

Bioinformatics analysis. TargetScan (http://www.targetscan. org/) and miRanda (http://www.microrna.org/microrna/home. do) were used to perform target prediction. The target genes predicted by TargetScan and miRanda were screened based on their scoring criteria. In the TargetScan algorithm, target genes with a context percentile $<50$ were excluded. In the miRanda algorithm, target genes with Max-Energy $>-10$ were excluded. The overlapping prediction results were selected as the candidate target genes of miR-22 $(24,25)$.

Statistical analysis. The data are presented as the mean \pm SEM. Statistical analysis was performed using SPSS 16.0 (SPSS, Inc.). Statistical comparisons between two groups were determined by two-tailed Student's t-test. One-way or two-way ANOVA with Bonferroni's post hoc test was performed for comparisons among multiple groups. $\mathrm{P}<0.05$ was considered to indicate a statistically significant difference.

\section{Results}

miR-22 is upregulated in osteoblasts treated with DEX. To confirm whether miR-22 is involved in DEX-induced osteoblast dysfunction, miR-22 expression in DEX-treated MC3T3-E1 cells was measured by qPCR. As presented in Fig. 1A, miR-22 expression levels in DEX-treated MC3T3-E1 cells were significantly higher compared with the control group, suggesting that miR-22 may serve a role in DEX-induced osteoblast dysfunction.

Transfection of miR-22 mimic aggravates, whereas miR-22 inhibitor attenuates DEX-induced dysfunction and apoptosis in osteoblastic MC3T3-E1 cells. The effects of miR-22 on DEX-induced osteoblast damage were further determined, and MC3T3-E1 cells were transfected with miR-22 mimic or inhibitor. As demonstrated in Fig. S1A and B, miR-22 levels significantly increased in the cells transfected miR-22 mimic compared with those transfected with control mimic; meanwhile, miR-22 levels significantly decreased in cells transfected with miR-22 inhibitor compared with those transfected with the miR-22 inhibitor control. As presented in Fig. 1B-E, DEX treatment and transfection with miR-22 mimic resulted in cell damage and apoptosis, as evidenced by significantly decreased cell viability and Bcl-2 protein levels as well as decreased ALP activity and the expression levels of apoptosis-related proteins cleaved caspase- 3 and Bax when compared with control groups. In addition, transfection with miR-22 mimic aggravated the DEX-induced osteoblast damage as the cell viability was significantly decreased, whereas ALP activity was significantly decreased in the cells transfected with miR-22 mimic compared with those transfected with the control group (Fig. 1B and C). miR-22 mimic also induced a significant increase in the expression levels of cleaved caspase-3 and Bax and decreased expression of Bcl-2 (Fig. 1D-F). By contrast, transfection with miR-22 inhibitor mitigated the osteoblast damage and apoptosis induced by DEX as evidenced by significantly increased cell viability, Bcl-2 levels and ALP activity as well as decreased expression levels of cleaved caspase- 3 and Bax.

A previous study demonstrated that p53 exerts a crucial role in inducing miR-22 transcription by binding to the promoter region of the miR-22 gene (26). In addition, p53 was reported to be associated with oxidative stress (27). Therefore, the present study determined whether the p53 signaling pathway was involved in the DEX-induced increased expression of miR-22. As presented in Fig. 2A and B, compared with controls, treatment with DEX resulted in significant increases in the protein expression levels of p53 in and its target $\mathrm{p} 21$, which were abrogated by the ROS scavenger NAC. Additionally, the DEX-induced increase in the expression levels of miR-22 was abolished by NAC and pifithrin- $\alpha$ (Fig. 2C). Collectively, these results suggested that the high expression levels of miR-22 in osteoblasts treated with DEX may be at least partly attributed to the ROS-induced the activation of the p53 signaling pathway.

miR-22 suppresses CAV3 expression in osteoblastic MC3T3-E1 cells. The present bioinformatics analysis identified one evolutionarily conserved miRNA recognition element that is partially complementary to miR-22 in the 3'-UTR of the CAV3 gene (Fig. 3C). As demonstrated in Fig. 3A and B, transfection with miR-22 mimic significantly decreased CAV3 expression 

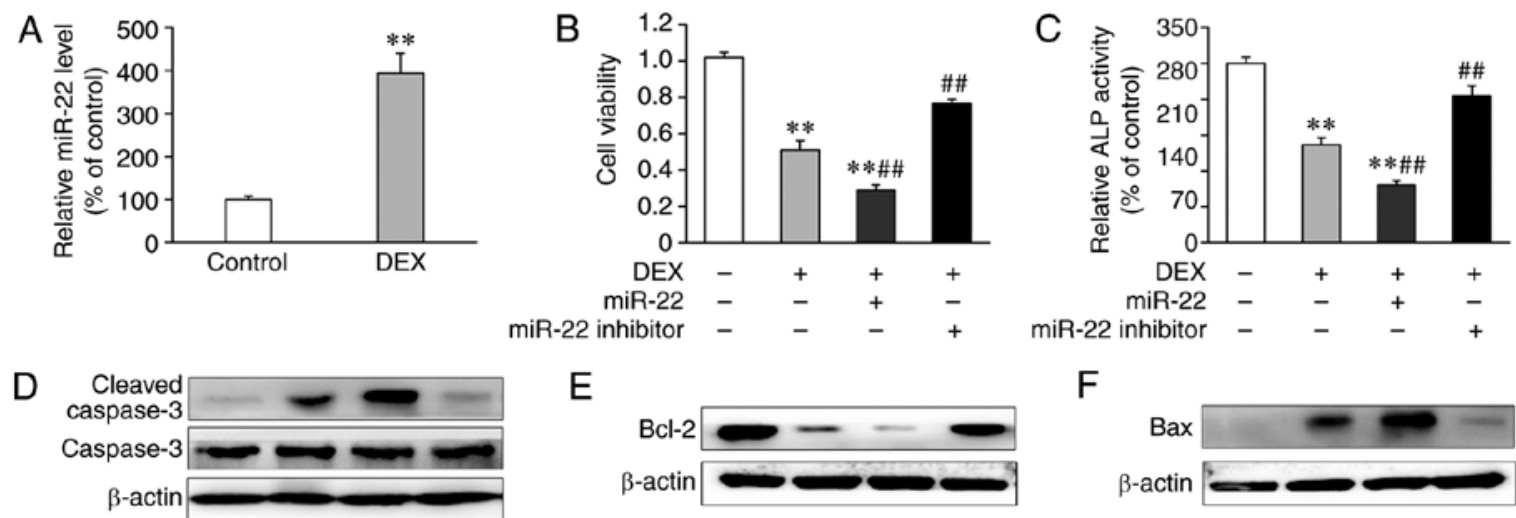

$\mathrm{F}$
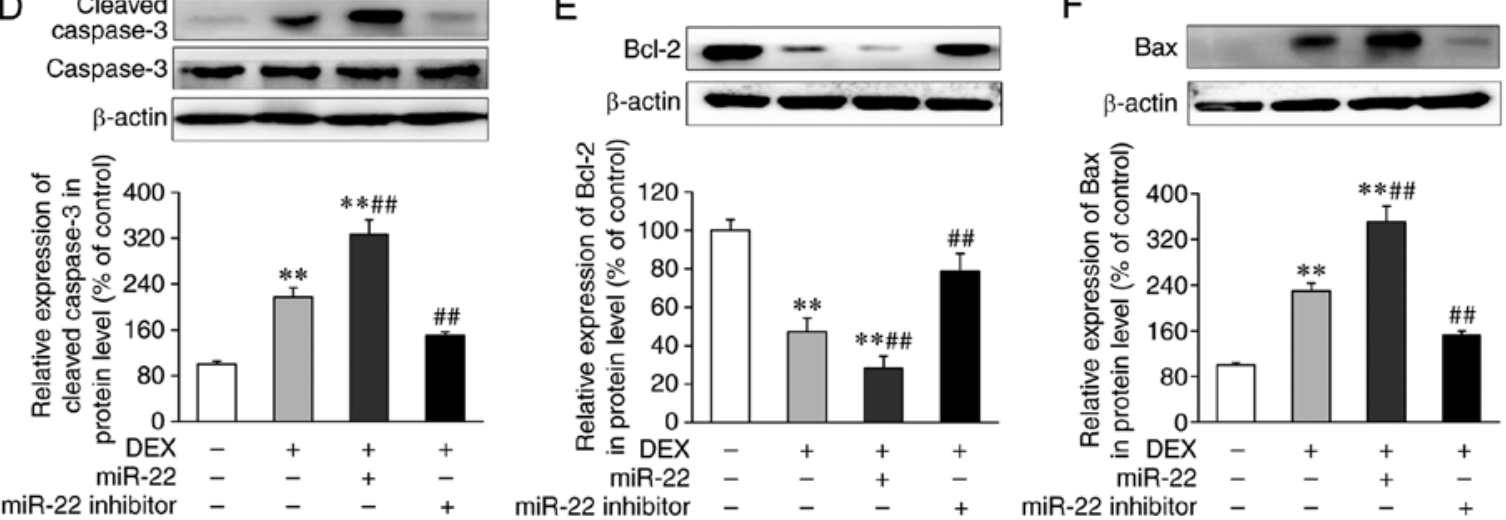

Figure 1. Upregulation of miR-22 contributes to DEX-induced osteoblastic MC3T3-E1 cell dysfunction and apoptosis. (A) Osteoblastic MC3T3-E1 cells were treated with $1 \mu \mathrm{M}$ DEX for $72 \mathrm{~h}$; cells treated with an equivalent volume of vehicle served as the control. Osteoblastic MC3T3-E1 cells were transfected with miR mimic and inhibitor control, miR-22 mimic or inhibitor for $24 \mathrm{~h}$, followed by DEX administration for $72 \mathrm{~h}$; (B) cell viability, (C) ALP activity and the protein levels of (D) caspase-3, Cleaved caspase-3, (E) Bcl-2 and (F) Bax were measured. Data are presented as the mean \pm standard error of the mean (n=4). ${ }^{* *} \mathrm{P}<0.01$ vs. control; ${ }^{\# \#} \mathrm{P}<0.01$ vs. DEX. miR, microRNA; DEX, dexamethasone; ALP, alkaline phosphatase.
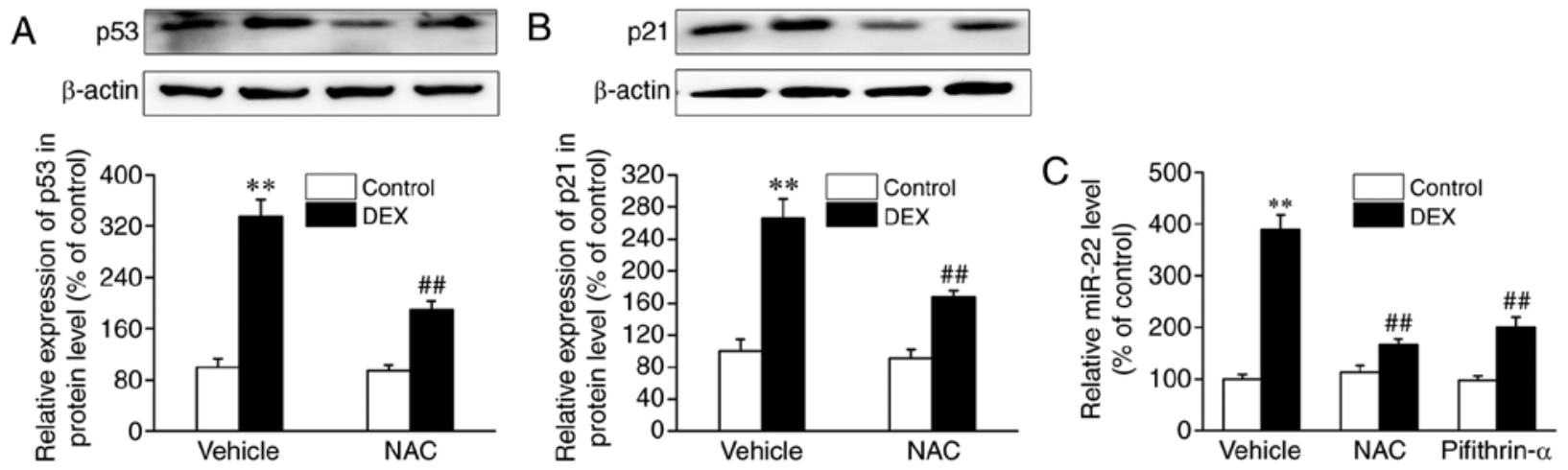

Figure 2. DEX-induced upregulation of miR-22 is abrogated by NAC and PFN- $\alpha$. Osteoblastic MC3T3-E1 cells were administrated with vehicle or DEX for $24 \mathrm{~h}$, followed by vehicle or $1 \mathrm{mM} \mathrm{NAC}$ for another $48 \mathrm{~h}$. Western blot analysis was performed to determine the protein expression levels of (A) p53 and (B) p21. (C) Osteoblastic MC3T3-E1 cells were administrated with vehicle or DEX for $24 \mathrm{~h}$, followed by $1 \mathrm{mM} \mathrm{NAC}$ or $20 \mu \mathrm{M}$ PFN- $\alpha$ for another $48 \mathrm{~h}$. Reverse transcription-quantitative PCR was performed to assess the expression levels of miR-22. Data are presented as the mean \pm standard error of the mean $(\mathrm{n}=4)$. ${ }^{* *} \mathrm{P}<0.01$ vs. control + vehicle; ${ }^{\# \prime} \mathrm{P}<0.01$ vs. DEX + vehicle. miR, microRNA; DEX, dexamethasone; NAC, N-acetyl L-cysteine; PFN- $\alpha$, pifithrin- $\alpha$.

at the mRNA and protein levels compared with controls. In order to determine whether miR-22 directly affected CAV3 expression, CAV3 3'-UTR luciferase reporter constructs and miR-22 mimic were transfected into osteoblasts. The results revealed significantly lower luciferase activity in the group co-transfected with miR-22 mimic compared with the miR control group (Fig. 3D). By contrast, no decrease in luciferase activity was observed following co-transfection of miR-22 when the putative miR-22 binding sequence in the CAV3 3'-UTR luciferase reporter construct was mutated. These results demonstrated that CAV3 expression was repressed in osteoblasts by the binding of miR-22 to response elements in its 3'-UTR.
Silencing of CAV3 abolishes the protective effects of miR-22 inhibitor against DEX-induced cell dysfunction and apoptosis in osteoblastic MC3T3-E1 cells. Next, the effects of miR-22 on CAV3 protein expression levels were determined in osteoblasts treated with DEX. As presented in Fig. 4A, CAV3 protein expression levels were reduced in osteoblasts treated with DEX, and miR-22 inhibitor reversed DEX-suppressed CAV3 expression. In addition, as shown in Fig. S1C and D, an $80 \%$ reduction in CAV3 expression levels was induced by CAV3 siRNA in osteoblasts compared with the control siRNA group. The results also demonstrated that the upregulation of CAV3 expression induced by miR-22 inhibitor in osteoblasts subjected to DEX was abolished by CAV3 siRNA. Further 
A

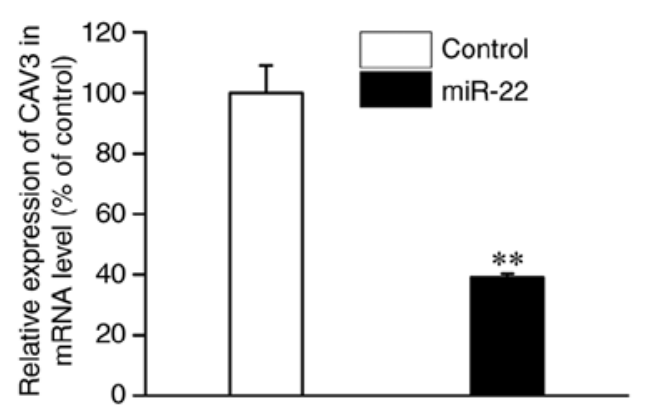

C

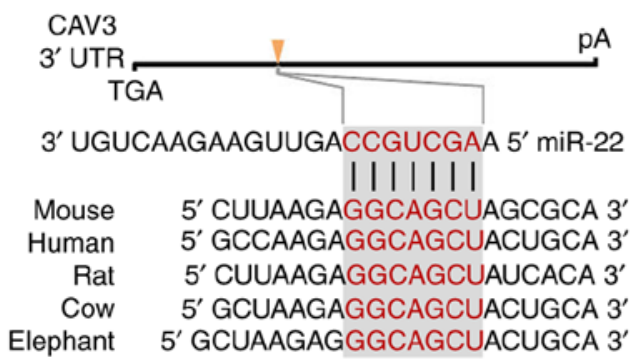

B
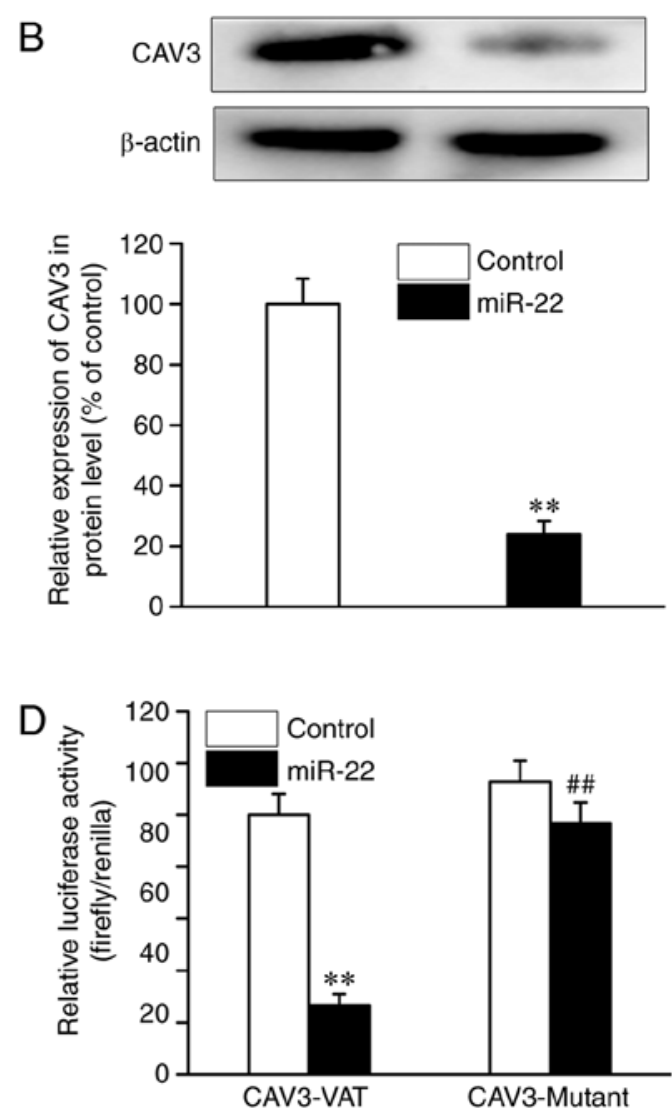

Figure 3. miR-22 represses CAV3 expression in osteoblastic MC3T3-E1 cells. Osteoblastic MC3T3-E1 cells were transfected with miR mimic control or miR-22 mimic for $24 \mathrm{~h}$. The (A) mRNA and (B) protein expression levels of CAV3 were measured by reverse transcription-quantitative PCR and western blot analysis, respectively, in osteoblastic MC3T3-E1 cells. (C) CAV3 was identified to contain highly conserved miR-22-mRNA interaction motifs within its 3'-UTR; the sequences of the WT and mutant 3'-UTR of CAV3 used for the luciferase reporter assay are presented. (D) Osteoblastic MC3T3-E1 cells co-transfected with WT or mutant CAV3 3'-UTR reporter plasmids and miR control or miR-22 mimic. Luciferase activity was measured using a dual luciferase assay after $24 \mathrm{~h}$. Data are presented as the mean \pm standard error of the mean $(\mathrm{n}=4) .{ }^{* * *} \mathrm{P}<0.01 \mathrm{vs}$. control; ${ }^{\# \#} \mathrm{P}<0.01 \mathrm{vs}$. miR-22 + CAV3-WT. miR, microRNA; CAV3, caveolin-3; UTR, untranslated region; WT, wild-type.

experiments were performed to confirm whether CAV3 was involved in the protective role of miR-22 inhibitor against DEX-induced osteoblast apoptosis. As presented in Fig. 4B-D, CAV3 siRNA abrogated the positive effects of miR-22 inhibitor against DEX-induced osteoblast apoptosis, as evidenced by significantly increased expression levels of cleaved caspase- 3 and Bax and the decreased expression levels of Bcl-2. In addition, CAV3 siRNA abrogated the positive effects of miR-22 inhibitor against DEX-induced osteoblast damage by significantly decreasing cell viability and increasing ALP activity (Fig. 4E and F).

\section{Discussion}

Osteoporosis is a common complication of long-term use of GCs and one of its characteristics is osteoblast dysfunction (5). A previous study has shown that miR-22 is a negative modulator of osteogenesis (13). The results of the present study also demonstrated that the levels of miR-22 expression were significantly upregulated in DEX-treated osteoblasts, and that upregulation of miR-22 may contribute to DEX-induced osteoblast dysfunction and apoptosis, as evidenced by decreased cell viability, Bcl-2 expression and ALP activity. In addition, the expression levels of apoptosis-related proteins cleaved caspase- 3 and Bax in cells transfected with the miR-22 mimic were increased compared with control cells, which were reversed by the miR-22 inhibitor. In addition, CAV3 was identified to be a target of miR-22 in osteoblasts, and CAV3 siRNA attenuated the protective effects of the miR-22 inhibitor on DEX-induced osteoblast damage and apoptosis.

The crucial roles of miRs, such as miR-2861, miR-17/20a, miR-221, miR-133a and miR-338-3p, in GIO have been validated in a number of studies $(11,12,28-31)$. Li et al (28) reported that miR-2861 exerts a physiological effect on osteoblast differentiation by targeting histone deacetylase 5 and contributes to primary osteoporosis in two related adolescents. Shi et al (11) demonstrated that miR-17/20a represses GC-induced osteoclast differentiation and function by targeting RANKL expression in osteoblastic cells. miR-22 is a widely expressed microRNA that is present at high levels in striated muscle tissues (31); thus, previous studies on miR-22 have focused on the cardiovascular system. Among them, Liang et al (13) demonstrated that miR-22 is involved in the negative regulation of osteogenesis by inhibiting the Wnt/ $\beta$-catenin pathway and osteoblast differentiation. Consistent with this, the results of the present study demonstrated that transfection with miR-22 mimic not only resulted in the damage, inhibition of differentiation and apoptosis in osteoblastic cells, but further aggravated DEX-induced osteoblast dysfunction and apoptosis. 
A
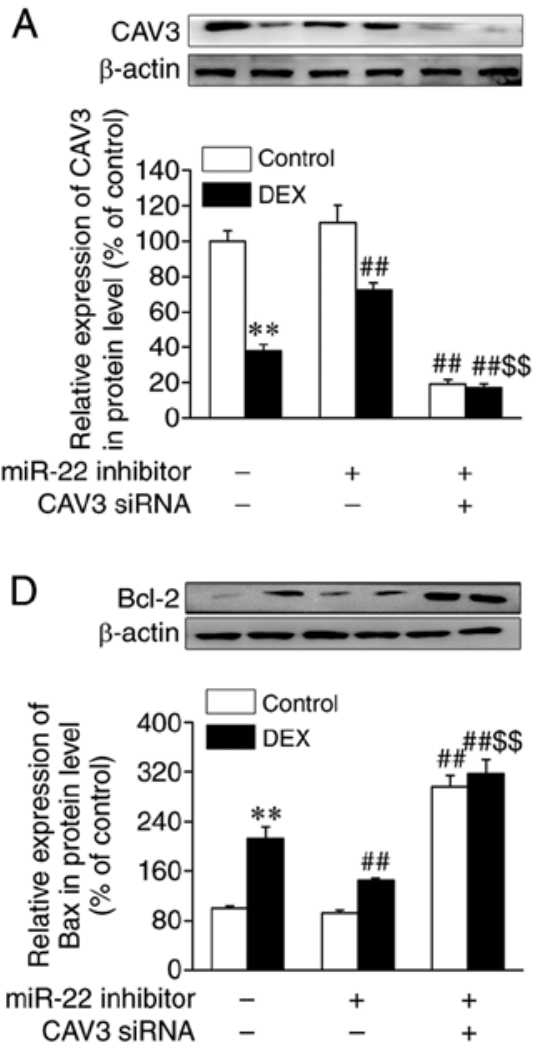

B
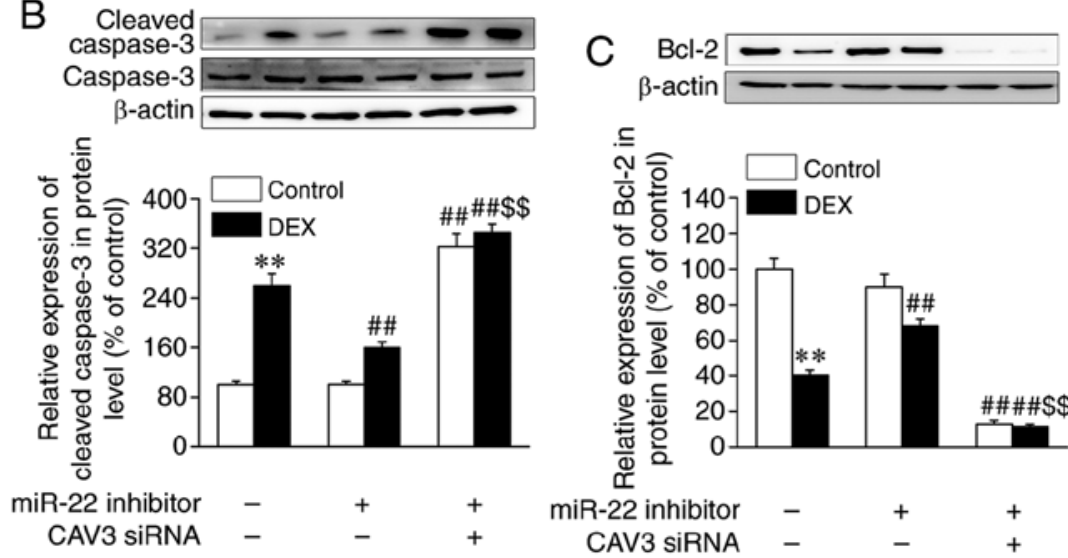

드

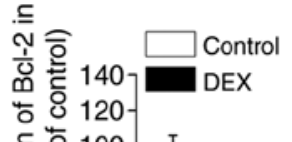

응요 $100-1$

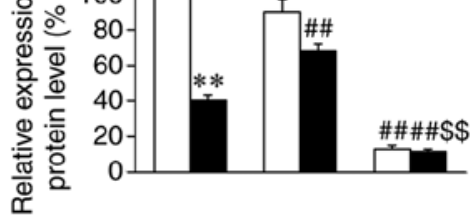

miR-22 inhibitor $\quad-\quad+\quad+$

CAV3 SiRNA - $\quad-\quad+$

Figure 4. Silencing of CAV3 abolishes the protective effects of miR-22 inhibitor against DEX-induced cell damage and apoptosis in osteoblastic MC3T3-E1 cells. Osteoblastic MC3T3-E1 cells were transfected with control or CAV3 siRNA for 24 h, followed by transfection with miR control or miR-22 inhibitor for $24 \mathrm{~h}$ and subsequent DEX treatment for the following $72 \mathrm{~h}$. Protein levels of (A) CAV3, (B) caspase-3, (C) Bcl-2, (D) Bax, (E) cell viability and (F) ALP activity were assessed. Data are presented as the mean \pm standard error of the mean $(n=4) .{ }^{* *} \mathrm{P}<0.01$ vs. control; ${ }^{\# \#} \mathrm{P}<0.01$ vs. $\mathrm{DEX}$; ${ }^{\$ \$} \mathrm{P}<0.01 \mathrm{vs}$. $\mathrm{DEX}+\mathrm{miR}-22$ inhibitor. miR, microRNA; CAV3, caveolin-3; DEX, dexamethasone; siRNA, small interfering RNA; ALP, alkaline phosphatase.

A previous study demonstrated that $\mathrm{p} 53$ exerts a crucial role in inducing miR-22 transcription by binding to the promoter region of the miR-22 gene (26). In addition, p53 was reported to be associated with oxidative stress (27). Li et al (32) demonstrated that DEX treatment induces p53 activation and inhibits the proliferation of MC3T3-E1 cells. Crochemore et al (33) also revealed that GC administration leads to rapid $\mathrm{p} 53$ nuclear translocation and promotes its transcriptional activity. The results of the present study demonstrated that the DEX-induced upregulation of p53 and p21 were mitigated by the ROS scavenger NAC. In addition, the DEX-induced increase in the expression levels of miR-22 was abolished by the ROS scavenger and pifithrin- $\alpha$. Collectively, these results demonstrated that high expression of miR-22 in osteoblasts treated with DEX may be at least partly attributed to the ROS-induced activation of the p53 signaling pathway.

CAV3, as a primary structural protein of the caveolae membrane domains, has been reported to be involved in maintaining the normal physiological cell structure and cell signaling; CAV3 is predominantly expressed in skeletal muscle, the diaphragm and the heart, and is selectively induced during the differentiation of skeletal C2C12 myoblasts $(14,34)$. Previous studies have demonstrated that CAV3 exerts a critical role in the progression of osteoporosis. Among them, Yang et al (14) reported that CAV3 upregulation enhances bone formation and inhibits the progression of osteoporosis by activating the Wnt signaling pathway in a rat model initially induced by means of ovariotomy, which suggested that CAV3 may be a potential target biomarker in osteoporosis treatment. Additionally, CAV3 has been reported to prevent apoptosis mediated by hypoxia or TNF- $\alpha$ treatment $(20,21)$. Consistent with these results, the results of the present study demonstrated that silencing of CAV3 by siRNA induced osteoblast cells damage and apoptosis, which were further exacerbated in DEX-treated osteoblasts.

Further experiments in the present study identified that CAV3 was a target of miR-22 in osteoblasts, which was consistent with the prediction of the bioinformatics analysis, as evidenced by the suppressed mRNA and protein expression levels as well as decreased luciferase activity of CAV3 3'-UTR luciferase reporter constructs following transfection with miR-22 mimic. Although Chen et al (35) did not report that CAV3 was a target of miR-22, they revealed that miR-22 was involved in myocardial ischemia and reperfusion by disrupting CAV-3/endothelial nitric oxide synthase signaling. In agreement with this, in the present study, the protective effects of miR-22 inhibitor against DEX-induced osteoblast damage and apoptosis was also reversed by CAV3 siRNA.

In conclusion, the results of the present study preliminary clarified that miR-22 may contribute to DEX-induced osteoblast differentiation inhibition and dysfunction as well as apoptosis by targeting CAV3 expression in osteoblastic cells. 


\section{Acknowledgements}

Not applicable.

\section{Funding}

This work was supported by the Key Incubation Project of Li Peng in the Science and Technology Department of Ningxia Medical University.

\section{Availability of data and materials}

The datasets used and/or analyzed during the current study are available from the corresponding author on reasonable request.

\section{Authors' contributions}

ZL performed the study design and prepared the manuscript. PL prepared the manuscript, collected data and performed statistical analysis. WM interpreted the data. SZ, LZ and ZC performed the literature search and experiments.

\section{Ethics approval and consent to participate}

Not applicable.

\section{Patient consent for publication}

Not applicable.

\section{Competing interests}

The authors declare that they have no competing interests.

\section{References}

1. van Staa TP, Leufkens HG, Abenhaim L, Begaud S, Zhang B and Cooper C: Use of oral corticosteroids in the United Kingdom. QJM 93: 105-111, 2000

2. Fardet L, Petersen I and Nazareth I: Prevalence of long-term oral glucocorticoid prescriptions in the UK over the past 20 years Rheumatology (Oxford) 50: 1982-1990, 2011

3. Overman RA, Yeh JY and Deal CL: Prevalence of oral glucocorticoid usage in the United States: A general population perspective. Arthritis Care Res (Hoboken) 65: 294-298, 2013.

4. Silverman S, Curtis J, Saag K, Flahive J, Adachi J, Anderson F, Chapurlat R, Cooper C, Diez-Perez A, Greenspan S, et al: International management of bone health in glucocorticoidexposed individuals in the observational GLOW study. Osteoporos Int 26: 419-420, 2015.

5. Ventura A, Brunetti G, Colucci S, Oranger A, Ladisa F, Cavallo L, Grano M and Faienza MF: Glucocorticoid-induced osteoporosis in children with 21-hydroxylase deficiency. Biomed Res Int 2013: 250462, 2013.

6. Delany AM, Dong Y and Canalis E: Mechanisms of glucocorticoid action in bone cells. J Cell Biochem 56: 295-302, 1994.

7. O'Brien CA, Jia D, Plotkin LI, Bellido T, Powers CC, Stewart SA, Manolagas SC and Weinstein RS: Glucocorticoids act directly on osteoblasts and osteocytes to induce their apoptosis and reduce bone formation and strength. Endocrinology 145: 1835-1841, 2004

8. Stamatopoulos B, Meuleman N, Haibe-Kains B, Saussoy P, Van Den Eric N, Michaux L, Heimann P, Martiat P, Bron D and Lagneaux L: microRNA-29c and microRNA-223 down-regulation has in vivo significance in chronic lymphocytic leukemia and improves disease risk stratification. Blood 113: 5237-5245, 2009.
9. Lee ST, Chu K, Im WS, Yoon HJ, Im JY, Park JE, Park KH, Jung KH, Lee SK, Kim M and Roh JK: Altered microRNA regulation in huntington's disease models. Exp Neurol 227: 172-179, 2011.

10. Du JK, Cong BH, Yu Q, Wang H, Wang L, Wang CN, Tang XL, Lu JQ, Zhu XY and Ni X: Upregulation of microRNA-22 contributes to myocardial ischemia-reperfusion injury by interfering with the mitochondrial function. Free Radic Biol Med 96: 406-417, 2016.

11. Shi C, Qi J, Huang P, Jiang M, Zhou Q, Zhou H, Kang H, Qian N, Yang Q, Guo L and Deng L: MicroRNA-17/20a inhibits glucocorticoid-induced osteoclast differentiation and function through targeting RANKL expression in osteoblast cells. Bone 68: 67-75, 2014.

12. Zhang Y, Gao Y, Cai L, Li F, Lou Y, Xu N, Kang Y and Yang H: MicroRNA-221 is involved in the regulation of osteoporosis through regulates RUNX2 protein expression and osteoblast differentiation. Am J Transl Res 9: 126-135, 2017.

13. Liang WC, Fu WM, Wang YB, Sun YX, Xu LL, Wong CW, Chan KM, Li G, Waye MM and Zhang JF: H19 activates Wnt signaling and promotes osteoblast differentiation by functioning as a competing endogenous RNA. Sci Rep 6: 20121, 2016.

14. Yang RB, Lin FF, Yang J, Chen B, Zhang MH, Lu QP, Xiao B, Liu Y, Zheng K and Qiu YR: Overexpression of CAV3 facilitates bone formation via the Wnt signaling pathway in osteoporotic rats. Endocrine 63: 639-650, 2019.

15. Chen F, Zhang L, OuYang Y, Guan H, Liu Q and Ni B: Glucocorticoid induced osteoblast apoptosis by increasing E4BP4 expression via up-regulation of Bim. Calcif Tissue Int 94: 640-647, 2014.

16. Zhang L, Yin HL, Jiao L, Liu TY, Gao YQ, Shao YC, Zhang YY, Shan HL, Zhang Y and Yang BF: Abnormal downregulation of caveolin-3 mediates the pro-fibrotic action of MicroRNA-22 in a model of myocardial infarction. Cell Physiol Biochem 45: 1641-1653, 2018

17. Galbiati F, Engelman JA, Volonte D, Zhang XL, Minetti C, Li M, Hou H Jr, Kneitz B, Edelmann W and Lisanti MP: Caveolin-3 null mice show a loss of caveolae, changes in the microdomain distribution of the dystrophin-glycoprotein complex, and t-tubule abnormalities. J Biol Chem 276: 21425-21433, 2001.

18. Gohel A, McCarthy MB and Gronowicz G: Estrogen prevents glucocorticoid-induced apoptosis in osteoblasts in vivo and in vitro. Endocrinology 140: 5339-5347, 1999.

19. Carotenuto F, Minieri M, Monego G, Fiaccavento R, Bertoni A, Sinigaglia F, Vecchini A, Carosella L and Di Nardo P: A diet supplemented with ALA-rich flaxseed prevents cardiomyocyte apoptosis by regulating caveolin-3 expression. Cardiovasc Res 100: 422-431, 2013.

20. Zhou Q, Peng X, Liu X, Chen L, Xiong Q, Shen Y, Xie J, Xu Z, Huang L, Hu J, et al: FAT10 attenuates hypoxia-induced cardiomyocyte apoptosis by stabilizing caveolin-3. J Mol Cell Cardiol 116: 115-124, 2018.

21. Tang YH, Yue ZS, Li GS, Zeng LR, Xin DW, Hu ZQ and Xu CD: Effect of $\beta$-ecdysterone on glucocorticoid-induced apoptosis and autophagy in osteoblasts. Mol Med Rep 17: 158-164, 2018.

22. Bowers GN Jr and McComb RB: A continuous spectrophotometric method for measuring the activity of serum alkaline phosphatase. Clin Chem 12: 70-89, 1966.

23. Livak KJ and Schmittgen TD: Analysis of relative gene expression data using real-time quantitative PCR and the 2(-Delta Delta C(T)) method. Methods 25: 402-408, 2001.

24. Meng SS, Wang H, Xue DB and Zhang WH: Screening and validation of differentially expressed extracellular miRNAs in acute pancreatitis. Mol Med Rep 16: 6412-6418, 2017.

25. Sun F, Yang X, Jin Y, Chen L, Wang L, Shi M, Zhan C, Shi Y and Wang Q: Bioinformatics analyses of the differences between lung adenocarcinoma and squamous cell carcinoma using the cancer genome atlas expression data. Mol Med Rep 16: 609-616, 2017.

26. Lin J, Huo R, Xiao L, Zhu X, Xie J, Sun S, He Y, Zhang J, Sun Y, Zhou Z, et al: A novel p53/microRNA-22/Cyr61 axis in synovial cells regulates inflammation in rheumatoid arthritis. Arthritis Rheumatol 66: 49-59, 2014.

27. Vurusaner B, Poli G and Basaga H: Tumor suppressor genes and ROS: Complex networks of interactions. Free Radic Biol Med 52: 7-18, 2012.

28. Li H, Xie H, Liu W, Hu R, Huang B, Tan YF, Xu K, Sheng ZF, Zhou HD, Wu XP and Luo XH: A novel microRNA targeting HDAC5 regulates osteoblast differentiation in mice and contributes to primary osteoporosis in humans. J Clin Invest 119: 3666-3677, 2009. 
29. Li Z, Zhang W and Huang Y: MiRNA-133a is involved in the regulation of postmenopausal osteoporosis through promoting osteoclast differentiation. Acta Biochim Biophys Sin (Shanghai) 50: 273-280, 2018.

30. Guo DW, Han YX, Cong L, Liang D and Tu GJ: Resveratrol prevents osteoporosis in ovariectomized rats by regulating microRNA-338-3p. Mol Med Rep 12: 2098-2106, 2015.

31. Christodoulou F, Raible F, Tomer R, Simakov O, Trachana K, Klaus S, Snyman H, Hannon GJ, Bork P and Arendt D: Ancient animal microRNAs and the evolution of tissue identity. Nature 463: 1084-1088, 2010.

32. Li H, Qian W, Weng X, Wu Z, Li H, Zhuang Q, Feng B and Bian Y: Glucocorticoid receptor and sequential P53 activation by dexamethasone mediates apoptosis and cell cycle arrest of osteoblastic MC3T3-E1 cells. PLoS One 7: e37030, 2012.
33. Crochemore C, Michaelidis TM, Fischer D, Loeffler JP and Almeida OF: Enhancement of p53 activity and inhibition of neural cell proliferation by glucocorticoid receptor activation. FASEB J 16: 761-770, 2002.

34. Tang Z, Scherer PE, Okamoto T, Song K, Chu C, Kohtz DS, Nishimoto I, Lodish HF and Lisanti MP: Molecular cloning of caveolin-3, a novel member of the caveolin gene family expressed predominantly in muscle. J Biol Chem 271: 2255-2261, 1996.

35. Chen Z, Qi Y and Gao C: Cardiac myocyte-protective effect of microRNA-22 during ischemia and reperfusion through disrupting the caveolin-3/eNOS signaling. Int J Clin Exp Pathol 8: 4614-4626, 2015.

This work is licensed under a Creative Commons Attribution-NonCommercial-NoDerivatives 4.0 International (CC BY-NC-ND 4.0) License. 